PROCEEDINGS OF THE

AMERICAN MATHEMATICAL SOCIETY

Volume 33, Number 2, June 1972

\title{
STRICT ESSENTIAL MINIMA
}

\author{
R. J. O'MALLEY 1
}

\begin{abstract}
A simple proof is given of the fact that the set of strict essential minima of a real function of $n$ variables is of measure zero. The proof uses only that a continuous function on a compact set has a maximum and the elementary fact, which seems to be new, that each set of positive measure contains a compact set which has positive upper density at each of its points.
\end{abstract}

In this paper, we establish a property of arbitrary real-valued functions defined on $E^{n}$. It is a simple fact that the set of strict relative minima of such a function is countable. If measure zero is used instead of countability, and if the strict essential minima are computed by the neglect of sets of outer metric density zero, then, as was shown in [1] and [2], a similar not so easy theorem holds. Our purpose is to obtain a proof of this result by augmenting the simple fact that a continuous function on a compact set has an absolute maximum with intuitively plain observations.

Indeed, if $f$ is measurable, its set, $M(f)$, of strict essential relative minima (i.e. sets of density zero are neglected) is measurable. If $M(f)$ has positive measure it has a compact subset $K$ of positive measure such that the density of $K$ is zero at none of its points and $f$ is continuous on $K$. The fact that $f$ has an absolute maximum on $K$ yields a contradiction. The case of an arbitrary function is obtained by use of the lower measurable boundary of $\mathrm{H}$. Blumberg [3].

Throughout this paper $D^{-}(A, x)\left(D_{-}(A, x)\right)$ will denote the ordinary upper (lower) metric density of $A$ at $x$ and $D_{S}(A, x)$ the upper symmetric density of $A$ at $x$, where

$$
\overline{D_{S}}(A, x)=\lim _{n \rightarrow \infty} \sup _{0<r<1 / n ; r \text { rational }}\left(\frac{|A \cap B(x, r)|}{|B(x, r)|}\right) .
$$

$|\cdot|$ denotes Lebesgue measure (outer measure if $A$ nonmeasurable) and $B(x, r)$ the closed ball of center $x$ and radius $r$.

Presented to the Society, November 20, 1971; received by the editors May 26, 1971. AMS 1970 subject classifications. Primary 28A20.

${ }^{1}$ Supported in part by National Science Foundation Grant NSF 9515 under the direction of Professor Casper Goffman.

(c) American Mathematical Society 1972 
We shall need the following notations and definitions.

(1) $A_{x}(f)=[y: f(y) \leqq f(x)]$,

(2) $B_{x}(f)=[y: f(y)>f(x)]$,

(3) $f$ is said to have a strict essential minimum at $x$ if $D^{-}\left(A_{x}, x\right)=0$,

(4) $M(f)=\left[x: D^{-}\left(A_{x}, x\right)=0\right]$.

We remark without proof that:

(5) $D_{S}^{-}(A, x)=0$ if and only if $D^{-}(A, x)=0$,

(6) by $(5), M(f)=\left[x: D_{S}^{-}\left(A_{x}, x\right)=0\right]$,

(7) if $D^{-}\left(A_{x}, x\right)=0$, then $D_{-}\left(B_{x}, x\right)=1$,

(8) if $A_{x}$ is measurable $D^{-}\left(A_{x}, x\right)=0$ if and only if $D_{-}\left(B_{x}, x\right)=1$.

We are now ready to state our theorem for measurable functions.

THEOREM. If $f$ is a measurable function, $|M(f)|=0$.

The proof of the theorem rests on the following two lemmas.

LEMMA 1. If $K^{0}$ is measurable and $\left|K^{0}\right|>a>0$, there is a compact $K$ such that

(i) $K \subset K^{0}$,

(ii) $|K| \geqq a$,

(iii) $D^{-}(K, x) \geqq \frac{1}{2}$ for every $x \in K$.

LEMMA 2. $M(f)$ is a measurable set.

For the sake of clarity, we first prove the theorem assuming the lemmas.

Suppose there is a positive number $a>0$ such that $|M(f)|>a$. Since $f$ is measurable, by Lusin's theorem and the lemmas, there is a compact set $K \subset M(f)$ satisfying (i), (ii), (iii) of Lemma 1 and on which $f$ is continuous. Therefore, there is an $x_{0} \in K$ such that:

$$
f(y) \leqq f\left(x_{0}\right) \text { for every } y \in K .
$$

This implies $K \subset A_{x_{0}}$ so that $D^{-}\left(A_{x_{0}}, x_{0}\right) \geqq \frac{1}{2}$, contradicting the fact that $x_{0}$ belongs to $M(f)$. This contradiction proves the theorem.

We will prove Lemma 1 for $K^{0} \subset E^{1}$, for $K^{0} \subset E^{n}, n \geqq 2$, the argument is the same. Clearly, there is no loss in generality in assuming that $K^{0}$ is a compact subset of $[0,1]$. For each $i=1,2, \cdots$, we let $I_{i j}=\left[(j-1) / 2^{i}\right.$, $\left.j / 2^{i}\right], j=1,2, \cdots, 2^{i}$, and $K_{m}^{1}$ represent the union of those sets, $K^{0} \cap I_{i j}$, $i=1, \cdots, m$, satisfying

$$
\left|K^{0} \cap I_{i j}\right| /\left|I_{i j}\right|>\frac{1}{2} \text {. }
$$

$K_{m}^{1}, m=1,2, \cdots$, is an increasing sequence of compact sets and it follows that if $x$ is any point of metric density 1 of $K^{0}$ then $x$ belongs to all but finitely many $K_{m}^{1}$. Therefore $\lim _{m \rightarrow \infty}\left|K_{m}^{1}\right|=\left|K^{0}\right|>a$, and we can find an $m_{1}$ such that $\left|K_{m_{1}}^{1}\right|>a$. We relabel $K_{m_{1}}^{1}=K^{1}$ and let $H_{1}$ denote the finite family, $I_{i j}$, satisfying $\left(^{*}\right)$ for $i \leqq m_{1}$. Replacing $K^{0}$ with $K^{1}$ we let $K_{m}^{2}, m>m_{1}$, be 
the union of those sets, $K^{1} \cap I_{i j}, i=m_{1}+1, \cdots, m$, satisfying

$$
\left|K^{1} \cap I_{i j}\right| /\left|I_{i j}\right|>\frac{1}{2} \text {. }
$$

There is an $m_{2}>m_{1}$ such that $\left|K_{m_{2}}^{2}\right|>a$ and

$$
\left|K_{m_{2}}^{2} \cap I_{i j}\right| /\left|I_{i j}\right|>\frac{1}{2} \text { for every } I_{i j} \in H_{1} .
$$

Relabel $K_{m_{2}}^{2}=K^{2}$ and let $H_{2}$ be the finite family of $I_{i j}$ satisfying $\left(^{* *}\right)$ for $m_{1}<i \leqq m_{2}$. Inductively, we proceed in the above fashion to obtain $K^{n} \subset K^{n-1}, n=1,2, \cdots, K^{n}$ compact, $\left|K^{n}\right|>a$, and a finite family $H_{n}$, such that the sets of intervals $H_{n}$ are pairwise disjoint and

$$
\left|K^{n} \cap I_{i j}\right| /\left|I_{i j}\right|>\frac{1}{2} \text { for every } I_{i j} \in H_{1} \cup H_{2} \cup \cdots \cup H_{n} .
$$

If we let $K=\bigcap_{n=1}^{\infty} K_{n}$ it is clear from the construction that $K$ satisfies (i), (ii). For (iii) let $x \in K$. For every $s$, there is $I_{s} \in H_{s}$ such that $x \in I_{s}$ and

Therefore

$$
\left|K^{n} \cap I_{s}\right| /\left|I_{s}\right|>\frac{1}{2} \text { for every } n \text {. }
$$

$$
\left|K \cap I_{s}\right| /\left|I_{s}\right| \geqq \frac{1}{2} \text { for every s. }
$$

Since $\left|I_{s}\right|$ tends to zero we have $D^{-}(K, x) \geqq \frac{1}{2}$ thus completing the proof of Lemma 1.

In order to prove Lemma 2, from (6) and the definition of $D_{S}^{-}\left(A_{x}, x\right)$ it suffices to show that if $f$ is a measurable function and $r$ is fixed, then $\left|A_{x} \cap B(x, r)\right|$ is a measurable function of $x$.

The proof of this fact is a variation of a proof in [4] and is included here for completeness.

Let $E=\left[x:\left|A_{x} \cap B(x, r)\right|<\lambda\right]$, we assume, as we may, that $\lambda>0$ and introduce the auxiliary function $\phi(x)$ given by

$$
\phi(x)=\text { g.l.b. }[c:|\{y: f(y) \leqq c\} \cap B(x, r)|>\lambda] .
$$

Clearly, $E=[x: f(x)<\phi(x)]$ and we will show $\phi(x)$ is a lower semicontinuous function, thereby establishing the iemma.

Let $x_{0}$ be fixed, $\varepsilon>0, \phi\left(x_{0}\right)=c_{0}$, by definition of $\phi\left(x_{0}\right)$ we have

$$
\left|\left\{y: f(y) \leqq c_{0}-\varepsilon\right\} \cap B\left(x_{0}, r\right)\right|=\lambda-d, \quad d>0 .
$$

By choosing $\delta>0$ small enough, we obtain

$$
\left|B(x, r) \backslash B\left(x_{0}, r\right)\right|<d \text { for every } x \in B\left(x_{0}, \delta\right),
$$

so that, for every $x \in B\left(x_{0}, \delta\right),\left|\left\{y: f(y) \leqq c_{0}-\varepsilon\right\} \cap B(x, r)\right|<\lambda-d+d=\lambda$ and $\phi(x) \geqq c_{0}-\varepsilon$. Since $\varepsilon>0$ is arbitrary, we have $\lim \inf _{x \rightarrow x_{0}} \phi(x) \geqq c_{0}=\phi\left(x_{0}\right)$ and $\phi$ is lower semicontinuous at $x_{0}$. This completes the proof of Lemma 2. We now extend the theorem to arbitrary functions. 
In [3], Blumberg defined the lower measurable boundary, $l(x)$, of a function $f(x)$ as l.u.b. $\left[c: D^{-}(\{y: f(y)<c\}, x)=0\right]$, and showed that $l$ is measurable and $f(x) \geqq l(x)$ almost everywhere.

Then $f(x) \leqq l(x)$ for every $x \in M(f)$. Accordingly, $l(x)=f(x)$ for almost everywhere $x$ in $M(f)$. We will let $M^{*}(f)$ be the set of all points $x \in M(f)$ which are points of outer metric density 1 of $M(f)$ and also $f(x)=l(x)$. Then $|M(f)|=\left|M^{*}(f)\right|$ where outer measure is now meant by $\mid \varphi$.

We consider $x_{0} \in M^{*}(f)$, by $(7), D_{-}\left(B_{x_{0}}, x_{0}\right)=1$, so that $\left[y: y \in M^{*}(f)\right.$ and $\left.f(y)>f\left(x_{0}\right)\right]$ has outer metric density 1 at $x_{0}$. Hence $C=\left[y: y \in M^{*}(f)\right.$, $\left.l(y)>l\left(x_{0}\right)\right]$ has outer metric density 1 at $x_{0}$. But $B_{x_{0}}(l)=\left[y: l(y)>l\left(x_{0}\right)\right]$ contains $C$ and is measurable.

By (8), this implies $D^{-}\left(A_{x_{0}}(l), x_{0}\right)=0$ and $x_{0} \in M(l)$. Therefore $M^{*}(f) \subset$ $M(l)$ which is of measure zero, by the theorem, and we have the following corollary.

COROLlaRY. If $f$ is arbitrary, $|M(f)|=0$.

\section{REFERENCES}

1. I. J. Good, The approximate local monotony of measurable functions, Proc. Cambridge Philos. Soc. 36 (1940), 9-13. MR 1, 206.

2. Á. Császár, Sur la structure des ensembles de niveau des fonctions réelles à deux variables, Acta Sci. Math. Szeged 15 (1954), 183-202. MR 16, 343.

3. H. Blumberg, The measurable boundaries of an arbitrary function, Acta Math. 65 (1935), 263-282.

4. J. C. Burkill and U. S. Haslam-Jones, The derivates and approximate derivates of measurable functions, Proc. London Math. Soc. 32 (1931), 346-355.

Department of Mathematics, Purdue University, Lafayette, Indiana 47907 